\title{
Storyboard writing and adult learners: A pragmatic approach
}

\begin{abstract}
A storyboard is essentially a timeline going from top to bottom, with the top occurring first. It is an expression of everything or even a rough sketch that will be contained in the program telling where the menu screens in a computer interface will look like, what pictures either still or moving will be seen when and for how long, what audio and text will accompany the images, either synchronously or hyperlinked or in other words allows us to see what the scene will look like. Typically, storyboards may be written documents and off-the-shelf storyboard pads for TV and video formats, adaptable for multimedia. The storyboard expresses, in one way or another, everything that can be seen or heard or experienced by the end-user of the multimedia program. It is more than a test-of-concept model, and just short of the final product specification document. Therefore, this paper investigates the content of discussion during storyboard writing and to investigate the meaning of the discussion. Five pairs of subjects were involved in this study. A task was given to each pair and the length of time for them to discuss about the layout and the content of the storyboard was 3 hours for each pair. The discussion will be taped and analyzed accordingly. The subjects were adult learners involved in a Language and Multimedia course ages between 33-40 years old, six female and four male. The subjects had no experience in storyboard writing. This paper uses the relevance theory (Sperber \& Wilson 1985), an inferential approach to pragmatics.
\end{abstract}

Keyword: Storyboard writing, pragmatic approach, adult learners 